\title{
RED TACTON \\ Human Area Networking
}

\author{
Prof. (Dr.) Sudan Jha \\ $P h D$ (Utkal University, Odisha, India) \\ PhD (OPJS University, Rajasthan, India) \\ Professor, School of Computer Engineering, KIIT University, Patia, BBSR, Odisha
}

\begin{abstract}
Technology is making many things easier; we can say that our concept is standing example for like that only. So far we have seen LAN, MAN, WAN, INTERNET \& many more but here a new concept of "RED TACTON" has been introduced which makes the human body as a communication network named by .... HAN (Human Area Network).

NTT lab from Japan is currently testing \& developing this revolutionary technology .Red Tacton is a new Human Area networking technology that uses the surface of the human body as a safe, high speed network transmission path. Red Tacton uses the minute electric field generated by human body as medium for transmitting the data. The chips which will be embedded in various devices contain transmitter and receiver built to send and accept data in digital format. In this paper we will discuss about red tacton, and its working. States, and applications of red tacton various fields. And we will compare our red tacton with the other technology for data transmission and know about human area network.
\end{abstract}

Keywords-UMTS (Universal Mobile Telecommunication Systems), International Telecommunications Union (ITU); Access Points; IMT-20001; 3G, HTTP, FTP; TELNET protocols, IEEE 802.1x protocol with EAP-TTLS; Mutual Authentication

\section{INTRODUCTION}

We may have imagined the feature as a place crawling with antennas and emitters, due to the huge growth of wireless communications. And it seems that the current means of transferring data might already have a very serious competitor none other than the human body.

Thus NTT labs from Japan has announced that is currently testing a revolutionary technology called "Red Tacton", which use the electric fields generated by the human body as medium for transmitting the data. The chips which will be embedded in various devices contain a transmitter and receiver built to send and accept data in digital format. The chips can take any type of file such as mp3 music file or mail and convert it in to the format that takes the form of digitals pulse that can be passed and read through a human being electric field. The chip in receiver devices reads these tiny changes and converts the file back into its original form.

\section{RED TACTON}

Red Tacton is a new Human Area Networking technology that uses the surface of the human body as a safe, high speed network transmission path. Red Tacton uses the minute electric field emitted on the surface of the human body. Technically, it is completely distinct from wireless and infrared .A transmission path is formed at the moment a part of the human body comes in contact with a Red Tacton transceiver. Physically separating ends the contact and thus ends communication Using Red Tacton, communication starts when terminals carried by the user or embedded in devices are linked in various combinations according to the user's Communication is possible using anybody surfaces, such as the hands, fingers, arms, feet, face, legs or torso.

Red Tacton works for natural physical movements 


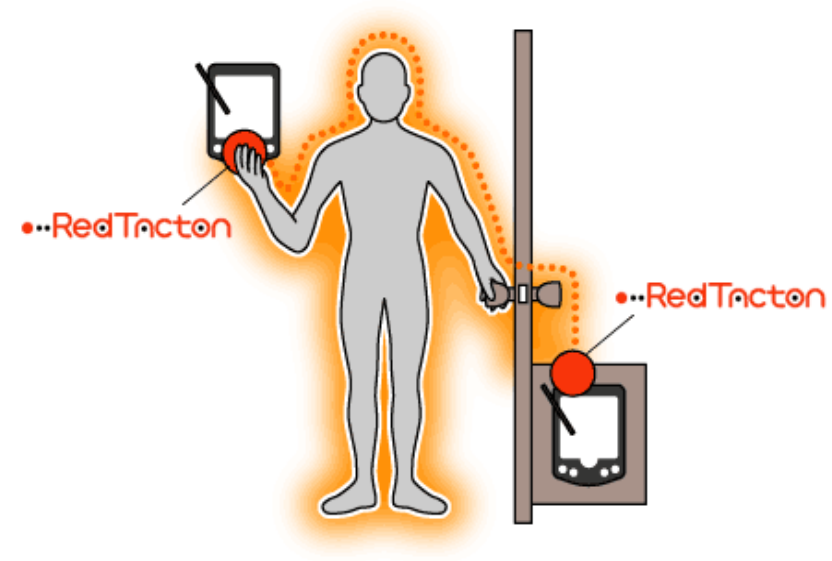

Fig 1: Overview of Red Tacton on HAN

- Registration of a user in a SN

- After a service request

- Location Update Request

- Attach Request

- Detach request

- Connection re-establishment request

Registration of a subscriber in a SN (Serving Network) typically occurs when the user goes to another country. The first time the subscriber then connects to the SN, he gets registered in the SN. Service Request is the possibility for higher-level protocols/applications to ask for AKA to be performed. E.g. performing AKA to increase security before an online banking transaction. The terminal updates the Home Location Register (HLR) regularly with its position in Location Update Requests. Attach request and detach request are procedures to connect and disconnect the subscriber to the network. Connection re-establishment request is performed when the maximum number of local authentications has been conducted.

\section{WORKING PRINCIPAL}

Using a new super-sensitive photonic electric field sensor, Red Tacton can achieve duplex

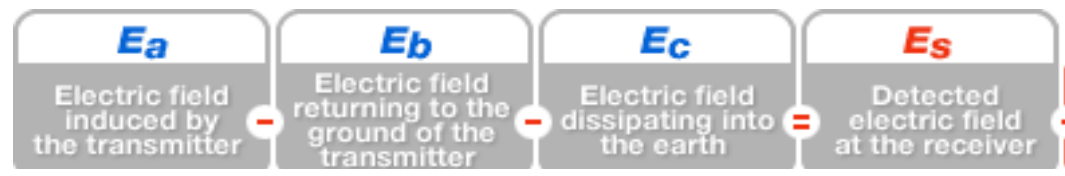

communication over the human body at a maximum speed of $10 \mathrm{mbps}$. The Red 


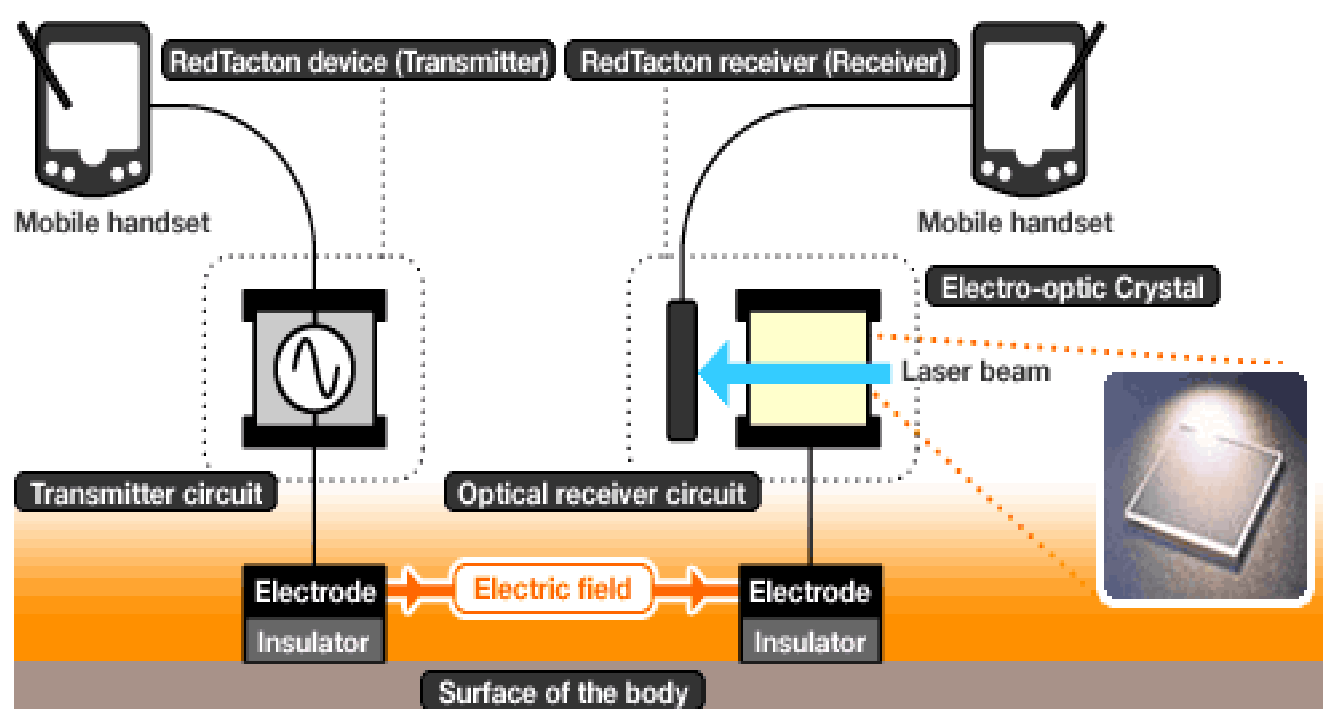

Fig 2 Human society is entering an era of ubiquitous computing.

Tacton transmitter induces a weak electric field on the surface of the body. The Red Tacton receiver senses principle that the optical properties of an electro-optic crystal can vary according to the changes of a weak electric field. Red Tacton detects changes in the optical properties of an electro-optic crystal using a laser and converts the result to an electrical signal in a optical receiver circuit.

The transmitter sends data by inducing fluctuations in the minute electric field on the surface of the human body. Data is received using a photonic electric field sensor that combines an electro-optic crystal and a laser light to detect fluctuations in the minute electric field. In addition to the WANs (Internet) and LANs, there are applications best served by Human Area Networks (HANs) that connect the last meter.

The naturally occurring electric field induced on the surface of the human body dissipates into the earth. Therefore, this electric field is exceptionally faint and unstable. The photonic electric field sensor developed by NTT enables weak electric fields to be measured by detecting changes in the optical properties of an electro-optic crystal with a laser beam.

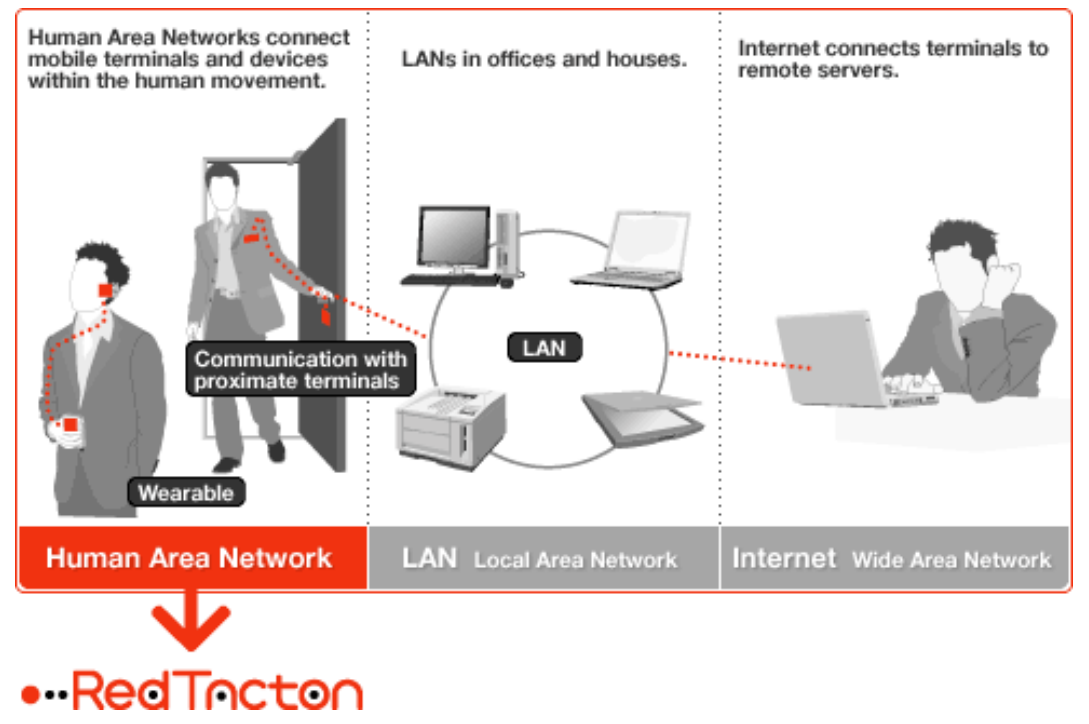

Fig:3 - Red Tacton has three main functional features 


\section{Human ArEa Network}

In addition to the WANs (Internet) and LANs, there are applications best served by Human Area Networks (HANs) that connect the last meter.

Human society is entering an era of ubiquitous computing, where everything is networked. By making Human Area Networks feasible, RedTacton will enable ubiquitous services based on human-centered interactions and therefore more intimate and easier for people to use.

\section{FeAtyre Of Red TACTON}

1. TOUCH: -

Communication with just a touch or step. Touching, gripping, sitting, walking, stepping and other human movements can be the triggers for unlocking or locking, starting or stopping equipment, or obtaining data. using RedTacton, communication starts when terminals carried by the user or embedded in devices are linked in various combinations through physical contact according to the human's natural movements.

\section{BROADBAND \& INTERACTIVE}

Duplex interactive communication is possible at a maximum speed of $10 \mathrm{Mbps}$. Because the transmission path is on the surface of the body, transmission speed does not deteriorate in congested areas where many people are communicating at the same time .Taking advantage of this speed, device drivers can be downloaded instantly and execute programs can be sent.

\section{ANY MEDIA}

In addition to the human body, various conductors and dielectrics can be used as transmission media. Conductors and dielectrics may also be

Used in combination

DIELETRICS

(Signals pass through materials)

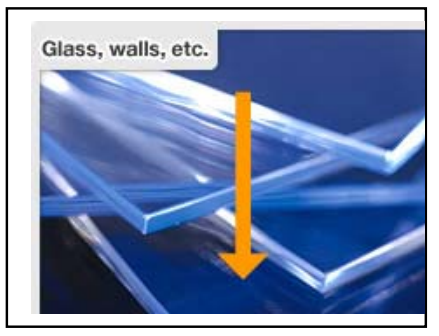

\section{CONDUCTORS}

(Signals travel along surface)

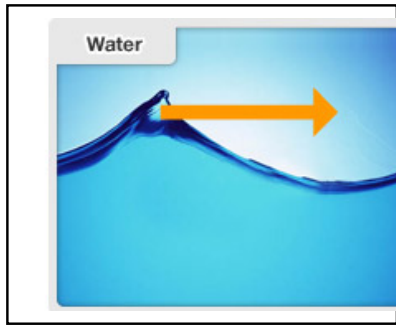

A communication environment can be created easily and at low-cost by using items close at hand, such as desks, walls, and metal objects. But there is one limitation on the length of the conductor to be propagated, on installation locations, and on the thickness of the dielectric to be passed through. 


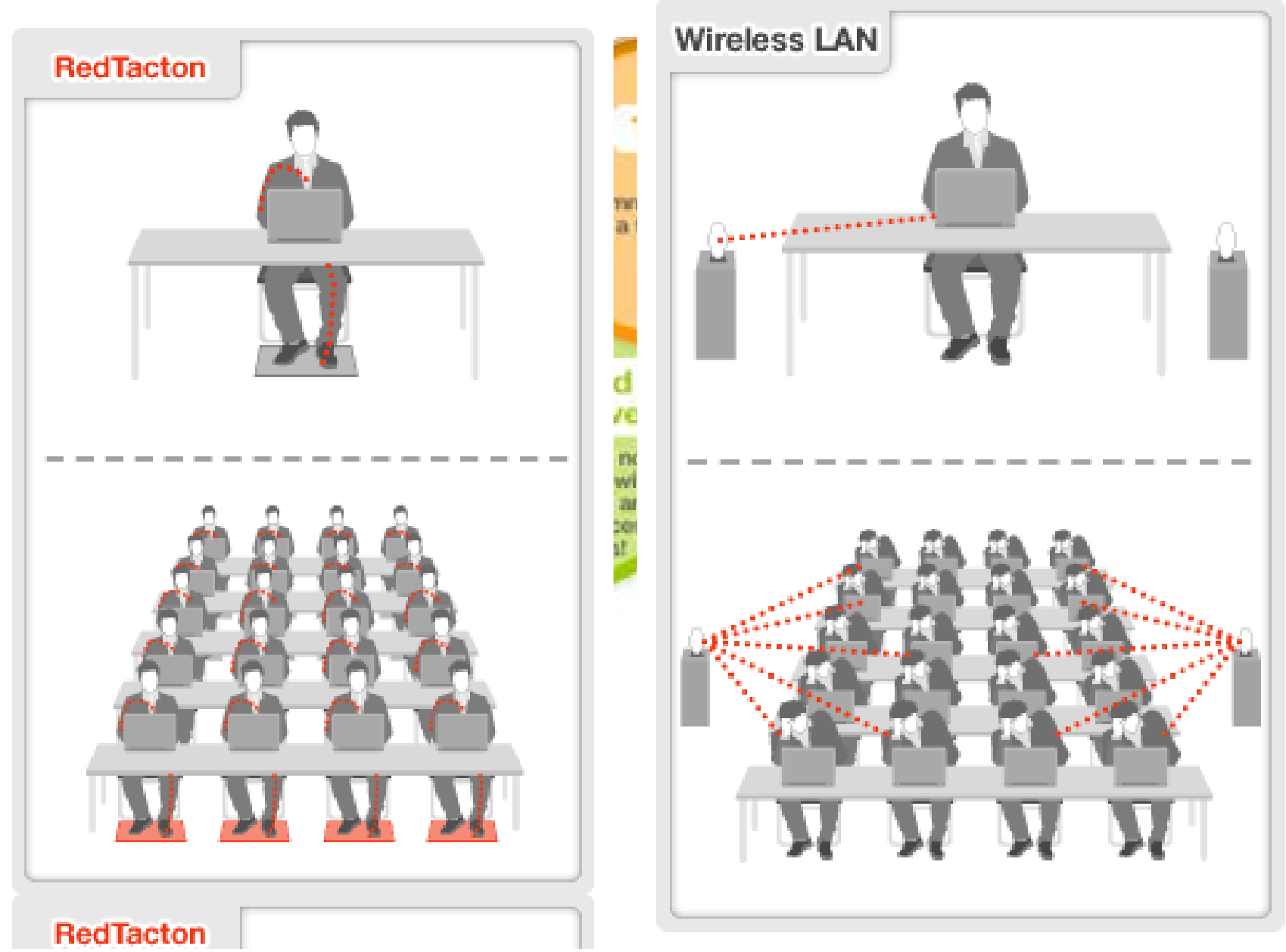

Fig: 3 Red Tacton has three main functional features.

A communication environment can be created easily and at low-cost by using items close at hand, such as desks, walls, and metal objects. But there is one limitation on the length of the conductor to be propagated, on installation locations, and on the thickness of the dielectric to be passed through.

i. The user and ownership network shared the system key K.

ii. The user trust the ownership network HE.

iii. The user's HE believes VLR can manage the information safely.

iv. The communication between HE and VLR is secure enough.

\section{APPPLICATION FIELDS}

Many application using Red Tacton are introduced. Some are:

i. An alarm sound automatically to avoid accidental medicine ingestion: -

Red tacton devices embedded medicine bottles transmit information on the medicines' attributes. If the user touches the wrong medicine, an alarm will trigger on the terminal he is carrying. The alarm sounds only if the user actually touches the medicine bottle, reducing false alarms common with passive wireless ID tags, which can trigger simply by proximity.

\section{ii. Touch advertising and receive information}


When a consumer stands in front of an advertising panel, advertising and information matching his or her attributes is automatically displayed. By touching or standing in front of items they are interested in, consumers can get more in-depth information.

iii. $\quad$ Touch a printer to print

Print out where you want just by touching the desired printer

with one hand and a PC or digital camera with the other hand to make the link Complicated configurations are reduced by downloading device drivers "at first touch".

iv. Instantaneous private network via personal handshake

By shaking hands, personal profile data can be exchanged between mobile terminals on the users. (Electronic exchange of business cards) Communication can be kept private using authentication and encryption technologies.

$v \quad$ Just touching a phone makes it your own

Your own phone number is allocated and billing commences. Automatic importing of personal address book and call history.

vi Just sitting in the seat triggers the car to load all its presets, just the way you like

The seat position and steering wheel height adjust to match the driver just by sitting in the car. The driver's home is set as the destination in the car navigation system . The stereo plays the driver's favorite song...

vii Connect to the network just by putting a lap-top on the table

An electrically conductive sheet is embedded in the table. A network connection is initiated simply by placing a lap-top on the table. Using different sheet patterns enables segmentation of the table into subnets.

viii Connect to the network just by putting a lap-top on the table

An electrically conductive sheet is embedded in the table. A network connection is initiated simply by placing a lap-top on the table. Using different sheet patterns enables segmentation of the table into subnets.

ix. Wireless headset

Red Tacton can carry music or video between headsets, mobile devices, mobile phones, etc. Users can listen to music from a Red Tacton player simply by putting on a headset or holding a viewer.

$x$. User verification and unlocking with just a touch

Carrying a mobile RedTacton-capable device in one's pocket, ID is verified and the door unlocked when the user holds the doorknob normally. Secure lock administration is possible by combining personal verification tools such as fingerprint ID or other biometric in the mobile terminal

\section{PROTOTYPE}

NTT has made three types of prototypes

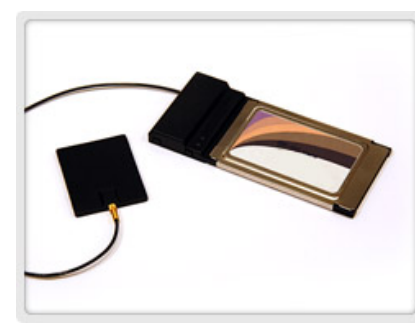

O RedTacton transceiver (PC card type)

a. Communicationspeed:10Mbps

b. Protocols : TCP/IP

c. Communication method :Half-duplex

d. Interface: PCMCIA 


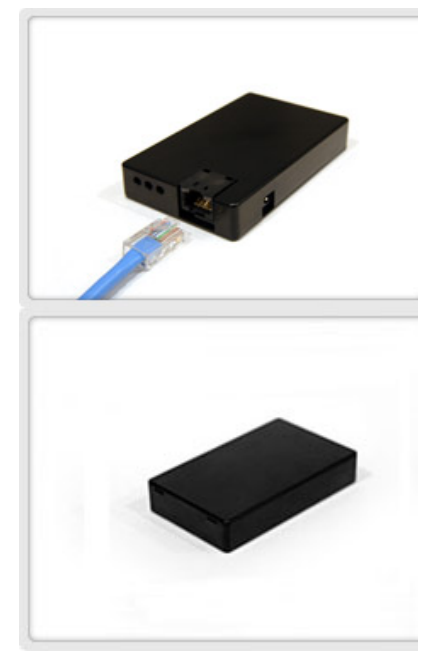

- RedTacton transceiver (hub type)

a. Communicationspeed:10Mbps

b. Protocols : TCP/IP

c. Communication method :Half-duplex

d. Interface: RJ45

\section{- RedTacton device (box type)}

(under constructions)

\section{COMPARISON WITH THE OTHER TECHNOLOGIES}

Please refer to the next page

\begin{tabular}{|c|c|c|c|c|c|c|c|}
\hline & & \multicolumn{4}{|c|}{ Wireless } & \multirow{2}{*}{$\begin{array}{c}\text { Infrared } \\
\text { Standard } \\
\text { data } \\
\text { communi- } \\
\text { cation }\end{array}$} & \multirow[b]{2}{*}{$\begin{array}{c}\text { Red } \\
\text { Tacton }\end{array}$} \\
\hline & Evaluation criteria & $\begin{array}{l}\text { Wirele } \\
\text { ss LAN }\end{array}$ & $\begin{array}{l}\text { Close } \\
\text { Range } \\
\text { wireless }\end{array}$ & $\begin{array}{c}\text { Contactless } \\
\text { IC } \\
\text { cards }\end{array}$ & $\begin{array}{l}\text { Passive } \\
\text { wireless } \\
\text { ID tag }\end{array}$ & & \\
\hline \multirow{3}{*}{ 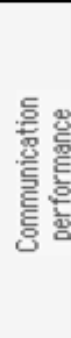 } & $\begin{array}{l}\text { Transfer speed(Can DVD- } \\
\text { quality images be sent?) }\end{array}$ & $\mathrm{E}$ & $P$ & $P$ & $P$ & $P$ & $\mathrm{E}$ \\
\hline & $\begin{array}{l}\text { Performance deterioration } \\
\text { during periods of ongestion } \\
\text { (Simultaneous use by many } \\
\text { people in small spaces) }\end{array}$ & $P$ & $P$ & $\mathrm{E}$ & $\mathrm{E}$ & $E$ & $E$ \\
\hline & $\begin{array}{l}\text { Duplex data transfer } \\
\text { (Interactive processing) }\end{array}$ & $\mathrm{E}$ & $\mathrm{E}$ & $\mathrm{E}$ & $\mathrm{P}$ & $\mathrm{E}$ & $E$ \\
\hline \multirow{3}{*}{ 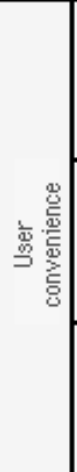 } & $\begin{array}{l}\text { Data configuration at } \\
\text { initiation of communications } \\
\text { (Registration of ID profiles, } \\
\text { etc) }\end{array}$ & E & $\mathrm{E}$ & $P$ & $P$ & $\mathrm{E}$ & E \\
\hline & $\begin{array}{l}\text { Tasks required at time of } \\
\text { each communication } \\
\text { (Adjustment of contacts and } \\
\text { optical axis) }\end{array}$ & $E$ & $\mathrm{E}$ & $P$ & E & $P$ & $E$ \\
\hline & $\begin{array}{l}\text { Synchronization with } \\
\text { user behavior } \\
\text { (Specification of } \\
\text { user positioning) }\end{array}$ & $P$ & $P$ & $\mathrm{E}$ & E & $P$ & $E$ \\
\hline
\end{tabular}

NOTE: E: Excellent P: Poor

\section{CONCLUSION}

So we conclude that, red tacton is the best technology when compared with other data communication technologies present today. In this technology there is no problem of hackers as 
our body is itself a media. If red tacton is introduced into cyber market it brings a marvelous \& tremendous change and will be adopted by many more people.

\section{REFERENCES}

[1] IEEE XPLORE "Education Technology and Computer Science, 2009. ETCS '09. First International Workshop on, 78 March 2009, Wuhan, Hubei.

[2] Engr.Mujtaba Hassan, Engr.Munaza Razzaq and Engr.Asim Shahzad,"Comprehensive Analysis of UMTS Authentication and Key Agreement" in International Journal of Computer and Network Security, Vol. 2, No.2, February 2010.

[3] K. Boman, G. Horn, P. Howard, and V. Niemi,"Umts Security”, October 2003

[4] Valtteri Niemi and Kaisa Nyberg,"UMTS Security", 2003.

[5] STEPHEN NORTHCUTT, JUdY NOVAK, Network Intrusion Detection

[6] CURRY, D., AND DEBAR, H. Intrusion Detection Message Exchange Format data model and Extensible Markup Language (XML) Document Type Definition

[7] W. R. CHESWICK, S. M. BELLOvin , "Firewalls and Internet Security : Repelling the wily hacker "

[8] "SunSHIELD Basic Security Module Guide" Sun MicroSystems Inc.

[9] Loris Degioanni, Fulvio Eisso, and Piero Viano, "Windump". http://netgroup- serv.polito.it/windump, erald Combs et al. "Ethereal". Available at http://www.ethereal.eom.

[10] "Etherpeek nx". http://www.wildpaekets.eom.

[11] "Gaim:A multi-protocol instant messaging (im) client", "http: //gaim.sourceforge.net/".

[12] "Ipv6: The Next Generation Internet!", "http://www.ipv6.org".

[13] Van Jacobson, Craig Leres, and Steven MeCanne, "tepdump : A Network Monitoring and Packet Capturing Tool". Available via anonymous FTP from ftp://ftp.ee.lbl.gov and www.tcpdump.org.

[14] Neeraj Kapoor. "Design and Implementation of a Network Monitoring Tool". Technical report, Department of Computer Science \& Engineering, IIT Kanpur, Apr 2001.

[15] http://www.cse.iitk.ac.in/research/mtech2000/Y011111.html.

[16] Steve MeCanne and Van Jacobson. "The BSD Packet Filter: A New Architecture for User-level Packet Capture". In Proceedings of USENIX Winter Conference, pages 259-269, San Diego, California, Jan 1993.

[17] "Network Associates Incorporated", http://www.sniffer.com.

[18] www.redtacton.com

[19] IEEE Internet Computing, special issue on Internet-Based Agents, vol. 1, 1997. 